\title{
Effects of Ovariectomy on Bone Metabolism and Bone Mineral Density in Spontaneously Diabetic Torii-Lepr ${ }^{f a}$ Rats
}

\author{
Shuichi KIMURA ${ }^{1) *}$, Tomohiko SASASE ${ }^{1)}$, Takeshi OHTA $^{1)}$ and Mutsuyoshi MATSUSHITA ${ }^{1)}$ \\ ${ }^{1)}$ Biological/Pharmaceutical Research Laboratories, Central Pharmaceutical Research Institute, Japan Tobacco Inc., Osaka 569-1125, \\ Japan
}

(Received 14 February 2011/Accepted 31 March 2011/Published online in J-STAGE 14 April 2011)

ABSTRACT. The Spontaneously Diabetic Torii-Lepr ${ }^{f a}$ (SDT- $f a / f a$ ) rat is a new model of obese type 2 diabetes. The female SDT- $f a / f a$ rat shows obesity, hyperglycemia and hyperlipidemia from a young age. However, it is not known whether diabetes and estrogen deficiency can lead to bone abnormalities in the female SDT- $f a / f a$ rat. The objective of the present study was to investigate the effects of ovariectomy (OVX) on bone metabolism and bone mineral density (BMD) in the female SDT-fa/fa rat. Female Sprague-Dawley rats were used as control animals. The BMDs of the whole tibia and fifth lumbar (L5) vertebral body were analyzed at 30 weeks after OVX. Serum osteocalcin, a bone formation marker, and urine deoxypyridinoline (DPD), a bone resorption marker, were sequentially analyzed before and at 5, 15 and 30 weeks after OVX. Serum osteocalcin and urine DPD levels were lower in SDT-fa/fa rats than in control rats before OVX. Both serum osteocalcin and urine DPD levels were elevated in control rats 5-30 weeks after OVX, but only the urine DPD levels were elevated in SDT- $f a / f a$ rats 5-30 weeks after OVX. SDT- $f a / f a$ rats showed a decrease in the BMDs of the whole tibia and L5 vertebral body compared with control rats. OVX decreased the BMDs of the whole tibia and L5 vertebral body in control rats, but not in SDT- $f a / f a$ rats. These data suggest that estrogen deficiency is not a risk factor for bone loss in type 2 diabetes mellitus. KEY WORDS: bone markers, bone mineral density, ovariectomy, rat, type 2 diabetes mellitus.

Diabetes mellitus is a risk factor for bone fracture. The risk of hip fracture is higher in diabetic patients than nondiabetic patients [7]. Bone mineral density (BMD) is a good predictor of bone strength because fracture risk increases with a decrease in BMD $[1,14]$. BMD is decreased in patients with type 1 diabetes [15] but is decreased [17], normal [15] or increased [3] in patients with type 2 diabetes. Unlike patients with type 1 diabetes, the relationship between BMD and fracture risk in patients with type 2 diabetes remains controversial.

Menopause is a major risk factor for bone fracture in women. Estrogen deficiency induces high bone turnover with an imbalance between bone resorption and bone formation in postmenopausal women and thereby a decrease in BMD leading to deterioration of the trabecular bone structure [13] and increased risk of bone fracture. Ovariectomy $(\mathrm{OVX})$ in rats is widely used as an appropriate model for postmenopausal osteoporosis in humans [8]. OVX has no effect on BMD in rats with type 1 diabetes [4], but its effect on BMD in rats with type 2 diabetes has not been reported.

The Spontaneously Diabetic Torii-Lepr ${ }^{f a}$ (SDT-fa/fa) rat, which was established by introducing the $f a$ allele of the Zucker fatty rat into the Spontaneously Diabetic Torii rat genome [10], eventually develops obesity, hyperglycemia, hyperlipidemia and diabetes-associated complications [6, $11,12]$. However, it is not known whether bone abnormalities attributable to diabetes and estrogen deficiency also

\footnotetext{
* Correspondence to: Kimura, S., Biological/Pharmaceutical Research Laboratories, Central Pharmaceutical Research Institute, Japan Tobacco Inc., 1-1 Murasaki-cho, Takatsuki, Osaka 569-1125, Japan.

e-mail: shuichi.kimura@jt.com
}

develop. The objective of the present study was to investigate the effects of OVX on bone metabolism and BMD in the female SDT-fa/fa rat. This study is the first report of the effect of ovariectomy on bone metabolism and BMD in type 2 diabetic rats.

\section{MATERIALS AND METHODS}

Animals: Female SDT-fa/fa rats from our colony were used. Age-matched female Sprague-Dawley rats (Charles River Laboratories Japan, Yokohama, Japan) were used as control animals. The rats were subjected either bilateral ovariectomy (OVX) or sham operation at 10 weeks of age. The rats were maintained at $23 \pm 3^{\circ} \mathrm{C}$ on a $12-\mathrm{hr} / 12$-hr lightdark cycle with ad libitum access to a standard diet (CRF-1; Oriental Yeast, Tokyo, Japan) and water. Thirty weeks after surgery, the rats were killed by exsanguination from the abdominal aorta under anesthesia by inhalation of diethyl ether. All animal procedures and protocols complied with the guidelines for animal experimentation set by the Ethics Committee for Animal Use at Japan Tobacco Inc.

Biochemical parameters: Biochemical parameters were evaluated before and at 5, 15 and 30 weeks after surgery (10, 15,25 and 40 weeks of age). Blood samples were collected from the tail vein. Four-hr urine samples were collected using metabolic cages. Glucose and creatinine were measured using commercial kits (Roche Diagnostics, Basel, Switzerland) and an automatic analyzer (Hitachi 7180; Hitachi, Tokyo, Japan). Insulin was measured using a commercial ELISA kit (Rat Insulin ELISA Kit; Morinaga Institute of Biological Science, Yokohama, Japan). Osteocalcin was measured using a commercial ELISA kit (Rat Osteocalcin 
[BGP] ELISA System; GE Healthcare, San Clemente, CA, U.S.A.). Deoxypyridinoline (DPD) was measured using a commercial EIA kit (Metra DPD EIA kit; Quidel, San Diego, CA, U.S.A.).

Bone mineral densitometry: The BMDs of the right whole tibia and fifth lumbar (L5) vertebral body were measured at 30 weeks after surgery ( 40 weeks of age) by quantitative computed tomography (QCT) using a LaTheta LCT-100A (Aloka, Tokyo, Japan) with a pixel size of $250 \times 250 \mu \mathrm{m}$ and a slice thickness is $1 \mathrm{~mm}$ for the right tibia and a slice thickness of $0.5 \mathrm{~mm}$ for the L5 vertebral body. The rats were anesthetized with pentobarbital $(40 \mathrm{mg} / \mathrm{kg}$ ) during measurement.

Statistical analysis: All data are presented as means $\pm \mathrm{SE}$. The statistical analyses were performed using SAS Version 8.2 and the SAS Preclinical Package Version 5.0 (SAS Institute Japan, Tokyo, Japan). Differences among all groups were tested by the following method. Bartlett's homogeneity of variance test was performed followed by Tukey's multiple comparison test for data with equal variances and the Steel-Dwass multiple comparison test for data with unequal variances. Differences were considered significant at $P<0.05$ (two-sided).

\section{RESULTS}

Body weight, serum glucose and insulin: Body weight was significantly higher in sham and OVX SDT- $f a / f a$ rats than in sham and OVX control rats (Fig. 1A). OVX control rats showed a significant increase in body weight compared with sham control rats 5-30 weeks after surgery, whereas there were no significant differences in body weight between sham and OVX SDT- $f a / f a$ rats. Serum glucose levels were significantly higher in sham and OVX SDT-falfa rats than in sham and OVX control rats (Fig. 1B). Serum insulin levels were significantly higher in sham and OVX SDT- $f a / f a$ rats than in sham and OVX control rats before and 5-15 weeks after surgery, but not significantly higher in sham SDT- $f a / f a$ rats than in OVX control rats at 15 weeks after surgery (Fig. 1C). Thus, OVX had no effect on serum glucose and insulin levels in both SDT- $f a / f a$ and control rats.

Bone metabolism: To evaluate the effect of OVX on bone metabolism in diabetic and control animals, serum osteocalcin, a bone formation marker, and urine DPD, a bone resorption marker, were measured (Table 1). Serum osteocalcin levels were significantly lower in SDT-falfa rats than in control rats before surgery and were significantly higher in OVX control rats than in sham control rats 5-30 weeks after surgery. On the other hand, serum osteocalcin levels were significantly higher in OVX SDT-falfa rats than in sham SDT- $f a / f a$ rats 5 weeks after surgery. Sham and OVX SDT$\mathrm{fa} / \mathrm{fa}$ rats showed significantly lower serum osteocalcin levels than sham and/or OVX control rats at all time points after surgery except for at 30 weeks. Urine DPD levels were significantly lower in sham and OVX SDT-falfa rats than in OVX control rats before surgery, significantly higher in
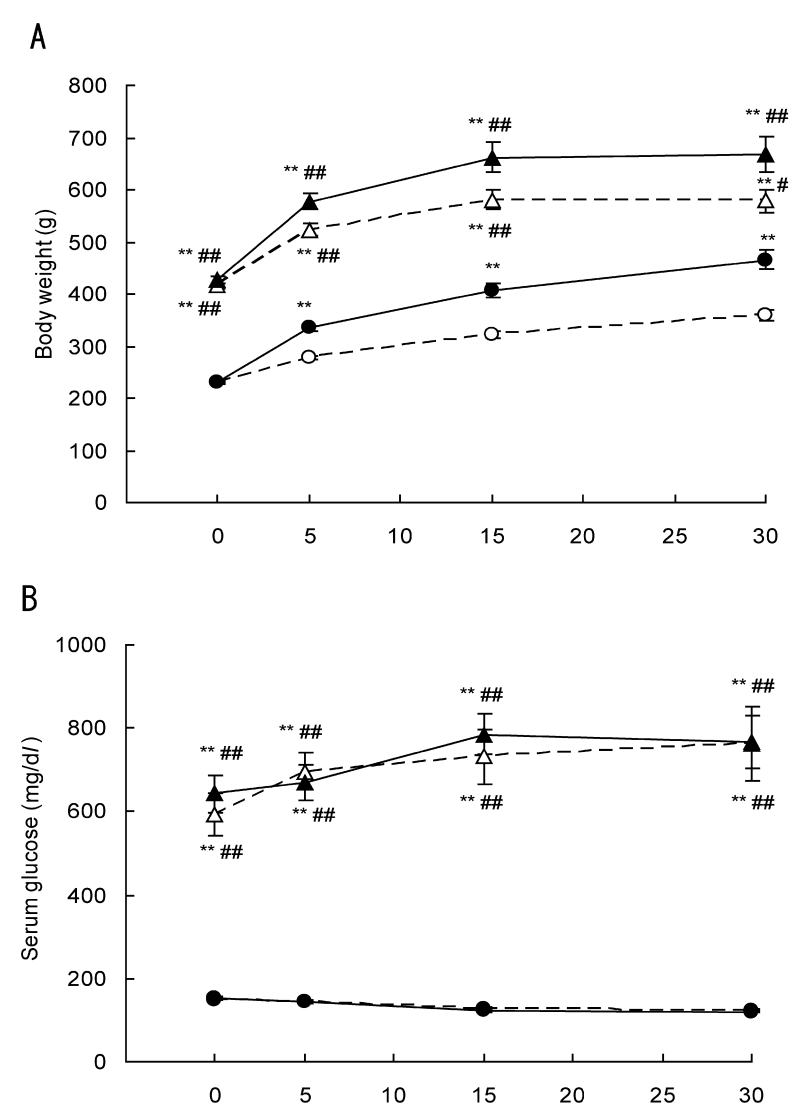

C

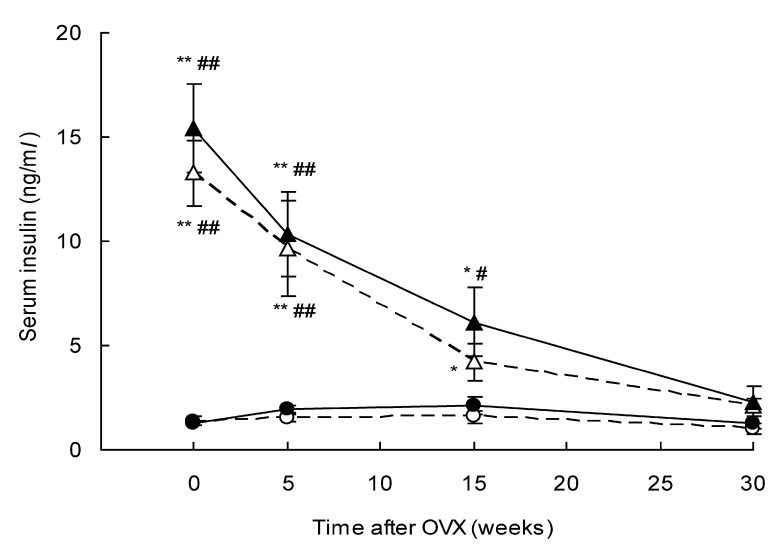

Fig. 1. Body weight (A), serum glucose (B) and serum insulin (C) in control and SDT-falfa rats. Symbols: sham control (open circle), OVX control (closed circle), sham SDT-fa/fa (open triangle) and OVX SDT-fa/fa (closed triangle) rats. Data represent means $\pm \mathrm{SE} ; \mathrm{n}=8-10$ per group. ${ }^{*}$ and $* *: P<0.05$ and $P<0.01$ vs. sham control rats, respectively. \# and \#\#: $P<0.05$ and $P<0.01$ vs. OVX control rats, respectively.

OVX control rats than in sham control rats 5-30 weeks after surgery and significantly higher in OVX SDT- $f a / f a$ rats than in sham SDT-falfa rats 5-30 weeks after surgery. Urine DPD levels in sham SDT- $f a / f a$ rats were significantly lower 
Table 1. Bone metabolism parameters

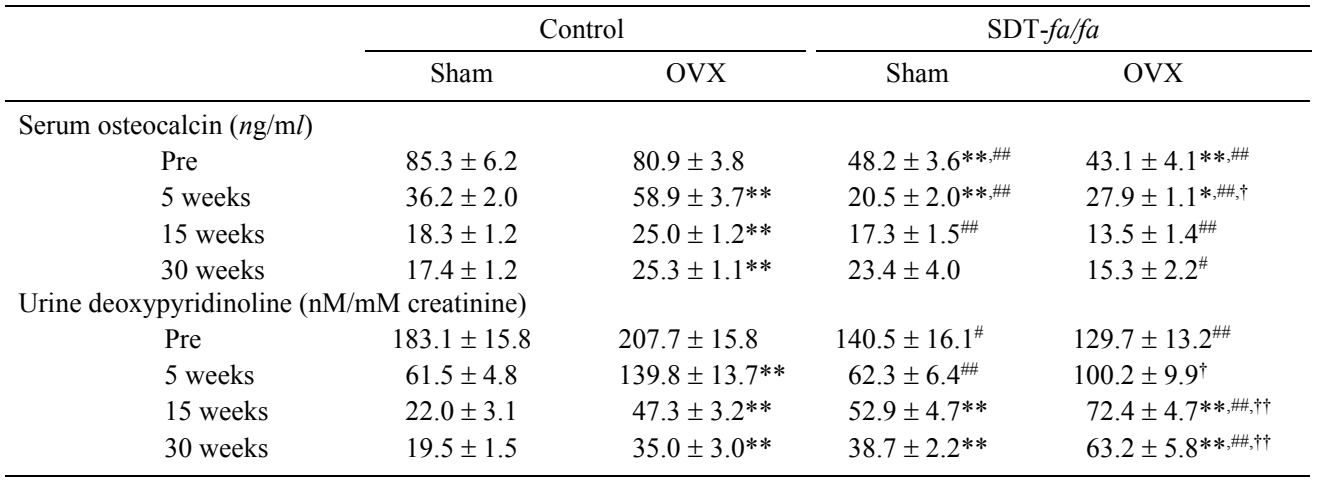

Data represent means $\pm \mathrm{SE} ; \mathrm{n}=8-10$ per group. ${ }^{*}$ and ${ }^{* *}: P<0.05$ and $P<0.01$ vs. sham control rats, respectively. \# and \#\#: $P<0.05$ and $P<0.01$ vs. OVX control rats, respectively. $\dagger$ and $\dagger \dagger: P<0.05$ and $P<0.01$ vs. sham SDT-falfa rats, respectively.

A

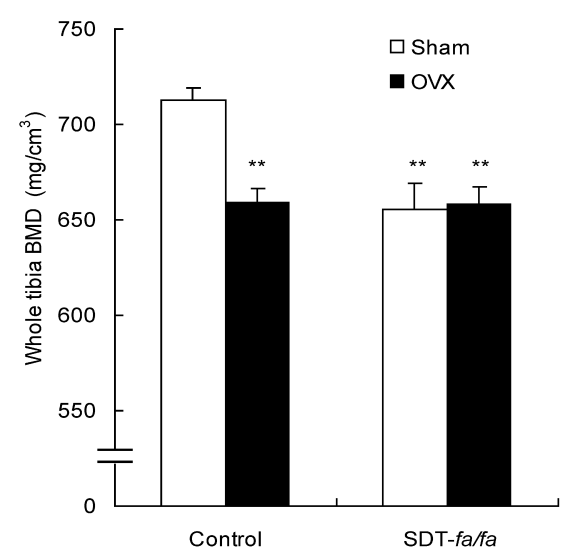

B

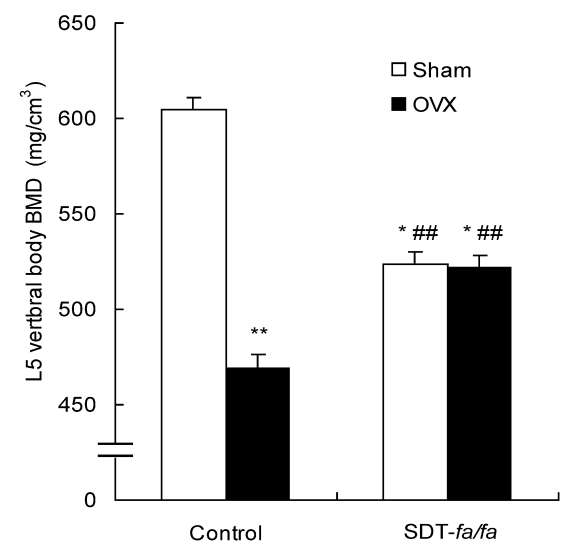

Fig. 2. Bone mineral densities of the whole tibia (A) and fifth lumbar (L5) vertebral body (B) in control and SDT $-f a / f a$ rats at 30 weeks after surgery (40 weeks of age). Data represent means \pm SE; $n=8-10$ per group. * and **: $P<0.05$ and $P<0.01$ vs. sham control rats, respectively. \#\#: $P<0.01$ vs. OVX control rats.

than in OVX control rats at 5 weeks after surgery, but significantly higher than in sham control rats $15-30$ weeks after surgery. OVX SDT-falfa rats showed significantly higher urine DPD levels than sham and OVX control rats 15-30 weeks after surgery.

$B M D$ : The BMDs of the whole tibia and L5 vertebral body were measured by QCT. The BMDs of the whole tibia and L5 vertebral body were significantly lower in OVX control rats than in sham control rats but were similar in sham and OVX SDT-falfa rats and significantly lower in sham and OVX SDT- $f a / f a$ rats than in sham control rats (Fig. 2A and $2 \mathrm{~B}$ ). On the other hand, the BMD of the L5 vertebral body was significantly higher in sham and OVX SDT-fa/fa rats than in OVX control rats (Fig. 2B).

\section{DISCUSSION}

The SDT- $f a / f a$ rat was established as a model of obese type 2 diabetes by Masuyama et al. [10]. The male SDT- $f a$ l $f a$ rat develops diabetes starting at 5 weeks of age and shows a cumulative incidence of $100 \%$ at 16 weeks of age. In this study, female sham SDT-falfa rats showed hyperglycemia with obesity starting at 10 weeks of age (Fig. 1A and 1B), with gradually decreasing serum insulin levels and gradually increasing serum glucose levels (Fig. 1B and 1C). These changes were also almost consistent with those in our previous report [6]. OVX had no effect on serum glucose and insulin levels in female SDT- $f a / f a$ rats as well as female control rats (Fig. 1B and 1C). Herrero et al. also showed no effect of OVX on serum glucose levels in streptozotocininduced diabetic rats [4]. These data suggest that OVX hardly affects glucose metabolism. 
The diabetes and obesity in female SDT- $f a / f a$ rats was associated with a decline in serum osteocalcin levels and resulted in low bone formation before and at 5 weeks after surgery (Table 1). Hyperglycemia is a negative factor for bone metabolism and causes osteoblast dysfunction. High glucose condition decreased cell proliferation and osteocalcin secretion in human osteoblast-like MG-63 cells [5]. Bone loss associated with low bone formation was observed in rats with diabetes $[4,16]$. On the other hand, urine DPD levels were higher in SDT-fa/fa rats than in control rats 1530 weeks after surgery (Table 1). High glucose condition facilitated osteoclastogenesis in murine bone marrow cells cocultured with a stromal cell line [5]. Kawashima et al. also reported that osteoclastogenesis and osteoclast activity were increased in type 2 diabetes model mice [9]. In addition, obesity impaired bone formation and decreased bone mass in growing rats [2]. Based on these data, it is considered that obese hyperglycemia reduces bone formation and elevates bone resorption, thereby causing severe bone loss in female SDT-fa/fa rats.

It is well known that OVX causes body weight gain, high bone turnover and bone loss. In this study, OVX control rats showed decreases in the BMDs of the whole tibia and L5 vertebral body (Fig. 2A and 2B) associated with high bone turnover (Table 1). On the other hand, the BMDs of the whole tibia and L5 vertebral body were similar in sham and OVX SDT- $f a / f a$ rats, although the urine DPD level continued to increase in OVX SDT- $f a / f a$ rats but not in sham SDT$f a / f a$ rats. A similar phenomenon was observed in other diabetic rat models. It was reported that OVX increased bone resorption without affecting lumbar vertebral BMD in female streptozotocin-induced diabetic rats [4]. Based on these data, it is considered that OVX in female diabetic rats accelerates bone resorption without causing a decrease in BMD. Because the reasons for the discrepancy between $\mathrm{BMD}$ and bone resorption in OVX SDT- $f a / f a$ rats is unclear, further studies are needed to elucidate this discrepancy.

The BMD in the L5 vertebral body was higher in OVX SDT- $f a / f a$ rats than in OVX control rats (Fig. 2B). OVX induces greater bone loss more prominently in cancellous bone than in cortical bone [18]. Since the lumbar vertebral body contains abundant cancellous bone, it is considered that OVX caused greater bone loss in the L5 vertebral body than in the whole tibia of control rats (Fig. 2A and 2B). On the other hand, OVX did not induce bone loss in SDT- $f a / f a$ rats, and consequently, it is considered that the BMD of the L5 vertebral body in SDT-fa/fa rats is higher than in control rats after OVX. A similar phenomenon is also observed in humans. Postmenopausal women with type 2 diabetes had higher lumbar spine BMDs than nondiabetic postmenopausal women [3]. Therefore, it is considered that the SDT$f a / f a$ rat may be a good model for bone abnormalities in postmenopausal women with type 2 diabetes.

In conclusion, female SDT- $f a / f a$ rats show decreases in the BMDs of the whole tibia and L5 vertebral body. OVX causes continuous elevations in urine DPD levels but has no effect on BMD in these rats. These data suggest that estro- gen deficiency is not a risk factor for bone loss in type 2 diabetes mellitus.

ACKNOWLEDGMENTS. The authors thank Mr. Nobuhiro Inaba and Ms. Sanae Nakamura (JT Creative Service) for long-term animal care.

\section{REFERENCES}

1. Broe, K. E., Hannan, M. T., Kiely, D. K., Cali, C. M., Cupples, L. A. and Kiel, D. P. 2000. Predicting fractures using bone mineral density: a prospective study of long-term care residents. Osteoporos. Int. 11: 765-771.

2. Chen, J. R., Lazarenko, O. P., Wu, X., Tong, Y., Blackburn, M. L., Shankar, K., Badger, T. M. and Ronis, M. J. 2010. Obesity reduces bone density associated with activation of PPARgamma and suppression of Wnt/beta-catenin in rapidly growing male rats. PLoS ONE 5: e13704.

3. Hadzibegovic, I., Miskic, B., Cosic, V., Prvulovic, D. and Bistrovic, D. 2008. Increased bone mineral density in postmenopausal women with type 2 diabetes mellitus. Ann. Saudi. Med. 28: $102-104$.

4. Herrero, S., Calvo, O. M., Garcia-Moreno, C., Martin, E., San Roman, J. I., Martin, M., Garcia-Talavera, J. R., Calvo, J. J. and del Pino-Montes, J. 1998. Low bone density with normal bone turnover in ovariectomized and streptozotocin-induced diabetic rats. Calcif. Tissue Int. 62: 260-265.

5. Inaba, M., Nishizawa, Y., Shioi, A. and Morii, H. 1997. Importance of sustained high glucose condition in the development of diabetic osteopenia: possible involvement of the polyol pathway. Osteoporos. Int. 7: S209-212.

6. Ishii, Y., Ohta, T., Sasase, T., Morinaga, H., Ueda, N., Hata, T., Kakutani, M., Miyajima, K., Katsuda, Y., Masuyama, T., Shinohara, M. and Matsushita, M. 2010. Pathophysiological analysis of female Spontaneously Diabetic Torii fatty rats. Exp. Anim. 59: 73-84.

7. Janghorbani, M., Van Dam, R. M., Willett, W. C. and Hu, F. B. 2007. Systematic review of type 1 and type 2 diabetes mellitus and risk of fracture. Am. J. Epidemiol. 166: 495-505.

8. Kalu, D. N. 1991. The ovariectomized rat model of postmenopausal bone loss. Bone Miner. 15: 175-191.

9. Kawashima, Y., Fritton, J. C., Yakar, S., Epstein, S., Schaffler, M. B., Jepsen, K. J. and LeRoith, D. 2009. Type 2 diabetic mice demonstrate slender long bones with increased fragility secondary to increased osteoclastogenesis. Bone 44: 648-655.

10. Masuyama, T., Katsuda, Y. and Shinohara, M. 2005. A novel model of obesity-related diabetes: introgression of the Lepr(fa) allele of the Zucker fatty rat into nonobese Spontaneously Diabetic Torii (SDT) rats. Exp. Anim. 54: 13-20.

11. Matsui, K., Ohta, T., Morinaga, H., Sasase, T., Fukuda, S., Ito, M., Ueda, M., Ogawa, N., Miyajima, K. and Matsushita, M. 2008. Effects of preventing hyperphagia on glycolipid metabolic abnormalities in Spontaneously Diabetic Torii fatty rats. Anim. Sci. J. 79: 605-613.

12. Matsui, K., Ohta, T., Oda, T., Sasase, T., Ueda, N., Miyajima, K., Masuyama, T., Shinohara, M. and Matsushita, M. 2008. Diabetes-associated complications in Spontaneously Diabetic Torii fatty rats. Exp. Anim. 57: 111-121.

13. Riggs, B. L. 1991. Overview of osteoporosis. West J. Med. 154: 63-77.

14. Schott, A. M., Cormier, C., Hans, D., Favier, F., Hausherr, E., Dargent-Molina, P., Delmas, P. D., Ribot, C., Sebert, J. L., 
Breart, G. and Meunier, P. J. 1998. How hip and whole-body bone mineral density predict hip fracture in elderly women: the EPIDOS Prospective Study. Osteoporos. Int. 8: 247-254.

15. Tuominen, J. T., Impivaara, O., Puukka, P. and Ronnemaa, T. 1999. Bone mineral density in patients with type 1 and type 2 diabetes. Diabetes Care 22: 1196-1200.

16. Verhaeghe, J., van Herck, E., Visser, W. J., Suiker, A. M., Thomasset, M., Einhorn, T. A., Faierman, E. and Bouillon, R. 1990. Bone and mineral metabolism in BB rats with long-term diabetes. Decreased bone turnover and osteoporosis. Diabetes
39: 477-482.

17. Yaturu, S., Humphrey, S., Landry, C. and Jain, S. K. 2009. Decreased bone mineral density in men with metabolic syndrome alone and with type 2 diabetes. Med. Sci. Monit. 15: CR5-9.

18. Zhang, Y., Lai, W. P., Leung, P. C., Wu, C. F. and Wong, M. S. 2007. Short- to mid-term effects of ovariectomy on bone turnover, bone mass and bone strength in rats. Biol. Pharm. Bull. 30: 898-903. 